\title{
Construction of a tunable promoter library to optimize gene expression in Methylomonas sp. DH-1, a methanotroph, and its application to cadaverine production
}

Hyang-Mi Lee ${ }^{1}$, Jun Ren ${ }^{1}$, Myeong-Sang Yu ${ }^{1}$, Hyunjoo Kim', Woo Young Kim¹, Junhao Shen ${ }^{1}$, Seung Min Yoo ${ }^{1}$, Seong-il Eyun ${ }^{2}$ and Dokyun $\mathrm{Na}^{1 *}$ (iD

\begin{abstract}
Background: As methane is 84 times more potent than carbon dioxide in exacerbating the greenhouse effect, there is an increasing interest in the utilization of methanotrophic bacteria that can convert harmful methane into various value-added compounds. A recently isolated methanotroph, Methylomonas sp. $\mathrm{DH}-1$, is a promising biofactory platform because of its relatively fast growth. However, the lack of genetic engineering tools hampers its wide use in the bioindustry.

Results: Through three different approaches, we constructed a tunable promoter library comprising 33 promoters that can be used for the metabolic engineering of Methylomonas sp. DH-1. The library had an expression level of $0.24-410 \%$ when compared with the strength of the lac promoter. For practical application of the promoter library, we fine-tuned the expressions of $c a d A$ and $c a d B$ genes, required for cadaverine synthesis and export, respectively. The strain with $\mathrm{P}_{\mathrm{rpmB}}$-cadA and $\mathrm{P}_{\text {DnaA }}$-cadB produced the highest cadaverine titre $(18.12 \pm 1.06 \mathrm{mg} / \mathrm{L})$ in Methylomonas sp. $\mathrm{DH}-1$, which was up to 2.8-fold higher than that obtained from a non-optimized strain. In addition, cell growth and lysine (a precursor of cadaverine) production assays suggested that gene expression optimization through transcription tuning can afford a balance between the growth and precursor supply.
\end{abstract}

Conclusions: The tunable promoter library provides standard and tunable components for gene expression, thereby facilitating the use of methanotrophs, specifically Methylomonas sp. DH-1, as a sustainable cell factory.

Keywords: Methylomonas sp. DH-1, Promoter library, Gene expression optimization, Cadaverine

\footnotetext{
*Correspondence: blisszen@cau.ac.kr

1 Department of Biomedical Engineering, Chung-Ang University, 84 Heukseok-ro Dongjak-gu, Seoul 06974, Republic of Korea

Full list of author information is available at the end of the article
}

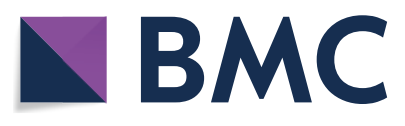

(c) The Author(s) 2021. Open Access This article is licensed under a Creative Commons Attribution 4.0 International License, which permits use, sharing, adaptation, distribution and reproduction in any medium or format, as long as you give appropriate credit to the original author(s) and the source, provide a link to the Creative Commons licence, and indicate if changes were made. The images or other third party material in this article are included in the article's Creative Commons licence, unless indicated otherwise in a credit line to the material. If material is not included in the article's Creative Commons licence and your intended use is not permitted by statutory regulation or exceeds the permitted use, you will need to obtain permission directly from the copyright holder. To view a copy of this licence, visit http://creativecommons.org/licenses/by/4.0/. The Creative Commons Public Domain Dedication waiver (http://creativeco mmons.org/publicdomain/zero/1.0/) applies to the data made available in this article, unless otherwise stated in a credit line to the data. 


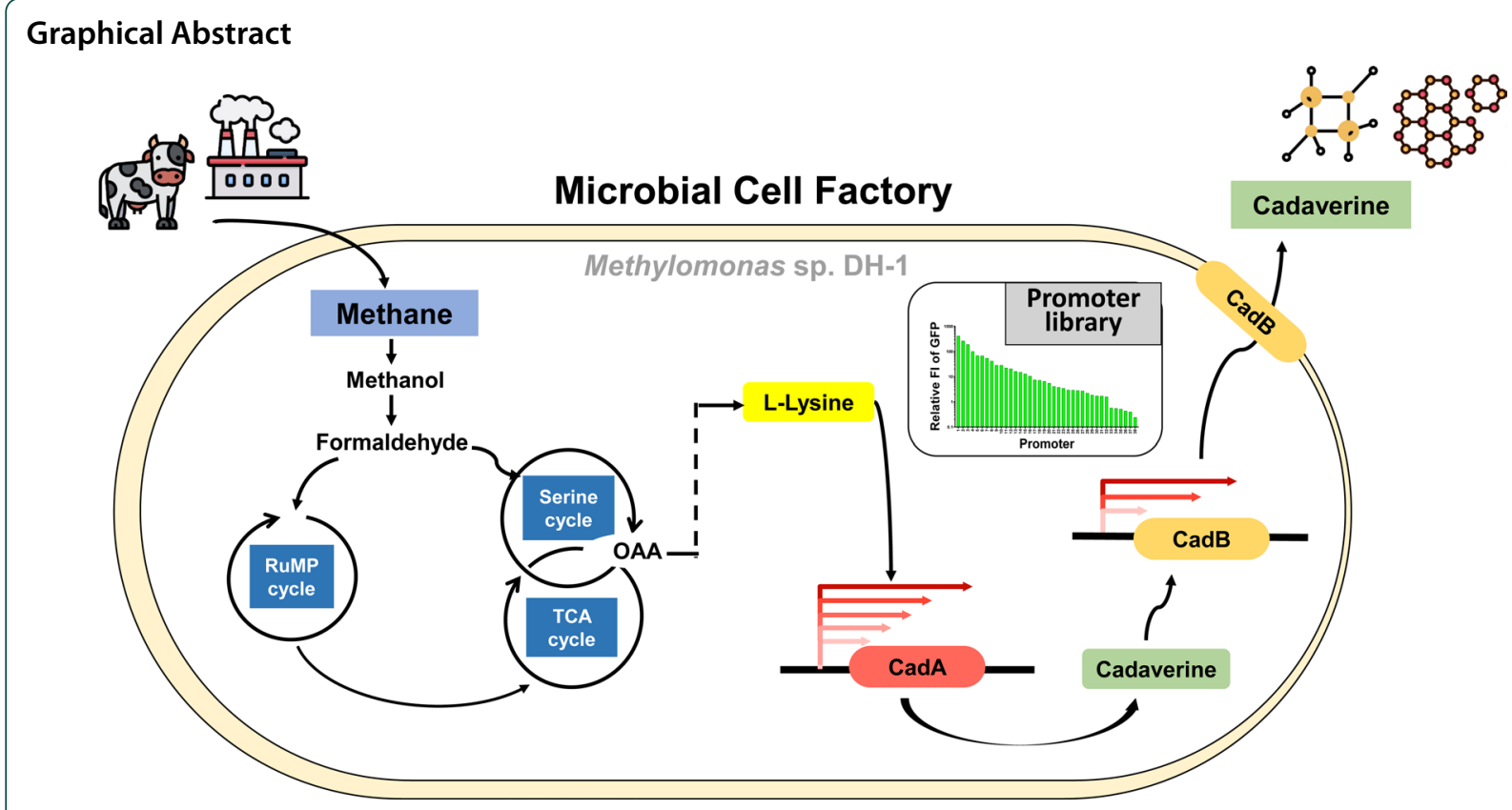

\section{Background}

Gene expression is a fundamental process of living organisms, specifically for processes, such as growth, metabolism, homeostasis, differentiation, and reproduction [1-3]. It has therefore been evolutionarily optimized for robust cellular functions. In cell engineering fields, such as synthetic biology and metabolic engineering, gene expression is fine-tuned for the robust operation of artificially designed genetic systems and enhanced production of a desired substance via manmade metabolic pathways [4-7].

In metabolic engineering, the overexpression of enzyme genes may fail to increase the production yield because cellular resources are excessively consumed for enzyme synthesis, resulting in decreased cell growth and thereby a decreased yield. In addition, the non-optimized expression of enzyme genes may facilitate the accumulation of toxic intermediate metabolites, which decreases the production yield. To resolve this problem, promoters with different strengths are widely employed to fine-tune the expression levels of enzyme genes [8,9].

Methylomonas sp. DH-1 is a newly isolated type I methanotrophic bacterium [10]. Its genomic and transcriptomic analyses have revealed that it possesses many genes that are involved in methane metabolism and secondary metabolite biosynthetic pathways, including the tricarboxylic acid (TCA) cycle, the ribulose monophosphate (RuMP) cycle, the Embden-Meyerhof-Parnas (EMP) pathway, the pentose phosphate (PP) pathway, the Entener-Doudoroff (EDD) pathway, and the methylerythritol 4-phosphate (MEP) pathway [10, 11]. Interestingly, the Methylomonas sp. DH-1 genome also contains a group of genes related to conventional type II methanotrophic metabolic pathways, such as tetrahydromethanopterin and tetrahydrofolate pathways [11]. Because of its high versatility, Methylomonas sp. DH-1 is a promising biocatalyst for efficiently converting methane into a variety of value-added products. As a result, the bacterium is used as a cell factory for producing various chemicals, such as methanol $(1.34 \mathrm{~g} / \mathrm{L})$ [12], 2-propanol $(0.424 \mathrm{~g} / \mathrm{L})$ [13], succinate $(0.195 \mathrm{~g} / \mathrm{L})$ [14], D-lactate $(1.19 \mathrm{~g} / \mathrm{L})$ [15], and acetone $(16.62 \mathrm{mM})$ [16].

Despite the versatility of Methylomonas sp. DH-1, however, the lack of well-established genetic engineering tools hampers its wide use as a cell factory platform. In an effort to facilitate genetic engineering of Methylomonas sp. DH-1, several synthetic tools for genetic manipulation have been developed over the past few years [1719]; however, there is an increased demand to develop a gene expression toolkit for the optimized metabolic engineering of Methylomonas sp. DH-1. A physicochemical transformation method using various chemicals (such as $\mathrm{RbCl}, \mathrm{LiAc}, \mathrm{CsCl}$, and $\mathrm{MgCl}_{2}$ ) and nanoparticles (such as sepiolite, gold(III) chloride, and chitosan) has been developed to increase the efficiency of transformation [17]. The restriction-modification system of Methylomonas sp. DH-1 has also been recently identified. The identified cytosine methyltransferase has been used for DNA methylation to protect DNA sequences, which increases the transformation efficiency [18]. More recently, a 
protein delivery system using cell-penetrating peptides was developed for manipulating the genome because no artificial episomal plasmids for the bacterium have yet been developed [19]. An application using a delivery system was used to excise an antibiotics resistance gene from the genome truncated by loxP sites. The delivery system directly transported Cre recombinase into the cytoplasm and successfully excised the target gene from the genome. Homologous recombination for gene disruption or integration and adaptive laboratory evolution for tolerance increase have also been performed as part of the metabolic engineering of Methylomonas sp. DH-1 $[14,15]$.

In this study, we construct a library of promoters that work in Methylomonas sp. DH-1 and can be used as components to fine-tune its gene expression. To demonstrate the applicability of the promoter library, we fine-tuned the expressions of two genes required for the biosynthesis and export of cadaverine, $c a d A$ and $c a d B$. Cadaverine is a bionylon monomer used for polyamide production. The developed tunable promoter library is a powerful tool for efficient expression optimization and may help advance the development of a sustainable chemical production platform via $\mathrm{C} 1$ assimilation.

\section{Results and discussion}

Construction of the promoter library for Methylomonas sp. DH-1

Among the various parameters that determine the expression level of a gene, transcription is the first step and the main target for gene regulation. As a result, promoters have been widely examined and used to control gene expression [20-22]. To identify potential promoters that can be used for gene expression optimization, we first utilized computational models to predict promoter sequence regions from the genomic sequence of Methylomonas sp. DH-1 [23, 24]. The computational prediction could not identify all the promoters of Methylomonas sp. DH-1 because the tools used have not been developed for Methylomonas sp. DH-1. To complement that, we also evaluated the promoters of $M$. trichosporium OB3b, a model organism of type II methanotroph [25], because Methylomonas sp. DH-1 also contains genes related to type II methanotroph [11] and they may share similar consensus promoter sequences. Therefore, the prediction was also performed on the genomic sequence of $M$. trichosporium OB3b to find more promoter candidates that could be applied to Methylomonas sp. DH-1. A total of 110 promoter candidates were predicted: 93 sequences from Methylomonas sp. DH-1 and 17 from M. trichosporium OB3b. When the predicted promoters were functionally categorized based on their downstream coding sequences, most of them can be grouped into genetic regulators, metabolism, and gene expression (Fig. 1A). Detailed information on the promoters is available in Additional files 2 and 3: Tables S1 and S2. In these tables, a promoter sequence has been defined as an upstream 100-bp sequence from the transcription start site, including -35 and -10 elements.

To find promoters with enough strength for efficient overexpression, the total cellular proteome of Methylomonas sp. DH-1 was quantitatively analysed by $2 \mathrm{D}$ gel electrophoresis (2D-PAGE) [26] (Fig. 1B; Additional file 1: Fig. S1). We chose five highly dense protein spots (indicated by arrows in Additional file 1: Fig. S1), which were then excised and digested with trypsin. The resulting peptides were analysed by matrix-assisted laser desorption/ionization-time of flight (MALDI-TOF) mass spectrometry, and the corresponding proteins were identified by peptide mass fingerprinting. The five highly expressed proteins were pyrroloquinoline quinone (PQQ)-dependent methanol dehydrogenase (encoded by the $m x a F$ gene), glutamine synthetase (encoded by the $g \ln A$ gene), elongation factor Tu (encoded by the tuf gene), transaldolase (encoded by the tal gene), and 3-hexulose-6-phosphate synthase (encoded by the hps gene). We extracted the $5^{\prime}$ upstream region of the protein-coding genes to obtain their promoter sequences, including -35 and -10 elements. The identified proteins and their promoter sequences are listed in Additional file 4: Table S3. Interestingly, none of the promoters identified by 2D-PAGE analysis overlapped with the promoters predicted by the computational tool.

In addition to the predicted and identified promoters, we included known promoters in our library: two M. trichosporium OB3b promoters (methanol dehydrogenase $(m x a F)$ and methane monooxygenase (pmoC)) and two E. coli promoters (lac and tac) (Fig. 1C). Finally, we compiled a library of 119 promoter candidates to construct a tunable promoter library (Fig. 1).

\section{Evaluation of promoter candidates in Methylomonas sp. DH-1}

To evaluate the promoter candidates, in addition to the promoters identified from 2D-PAGE analysis and the known promoters, we randomly selected several promoters from each category. The transcriptional activities of 38 out of 119 candidate promoters in Methylomonas sp. DH-1 were quantitatively measured. For the strength measurement, a plasmid harbouring the green fluorescent protein $(g f p)$ gene under the control of a promoter candidate was constructed.

Protein production level is determined not only by promoter strength but also by translational efficiency. To exclude the effect of translation on protein production, we used the same 50-nt long UTR, SD, and GFP coding 


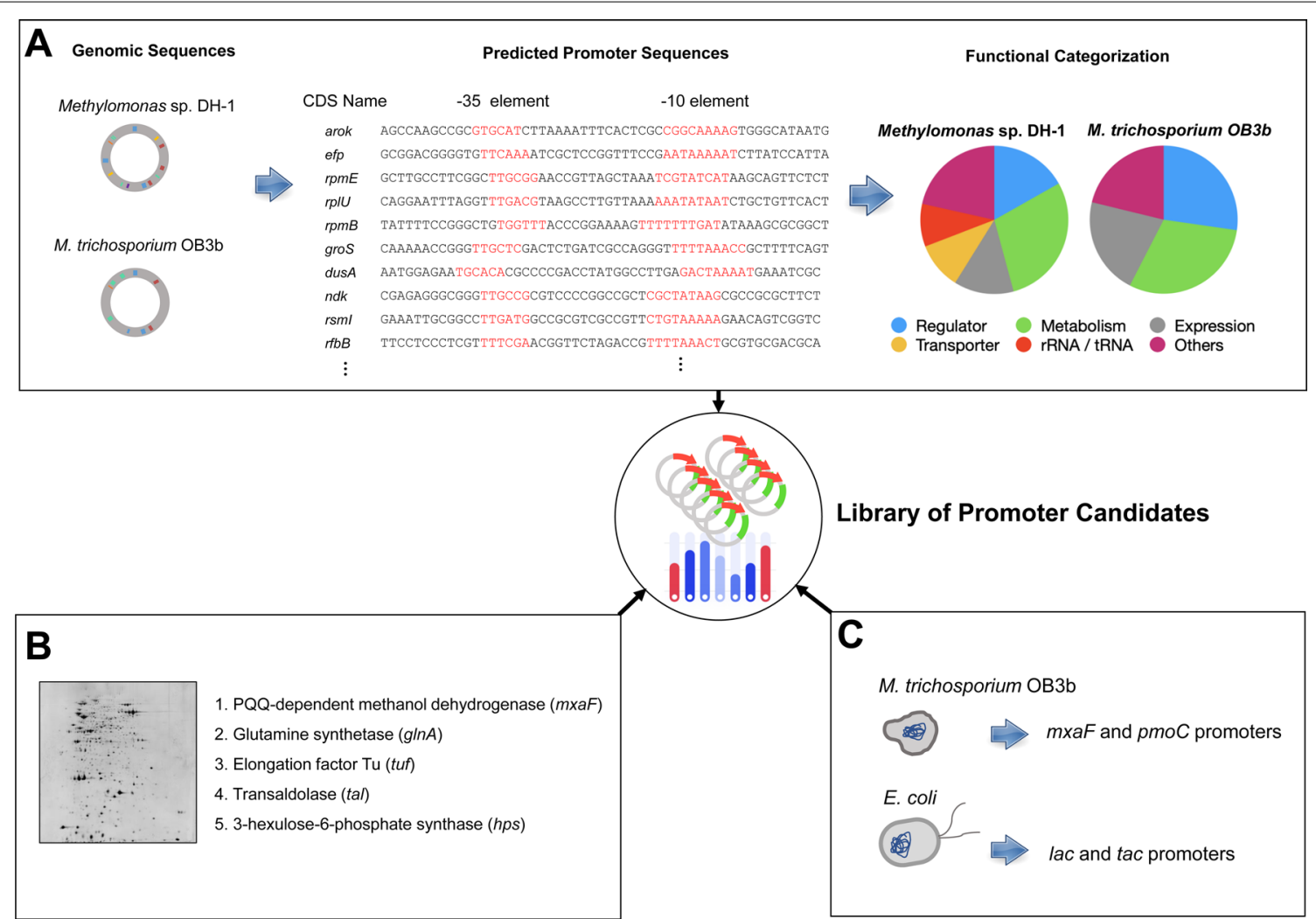

Fig. 1 Construction of a library of promoter candidates in Methylomonas sp. DH-1. A In silico approach to predict promoters from the genomic sequences of Methylomonas sp. DH-1 and M. trichosporium OB3b and the functional classification of the coding sequences under the control of the predicted promoters. B 2D-PAGE approach to identify strong native promoters that allow for the overexpression of Methylomonas sp. DH-1. C Addition of four well-known promoters of M. trichosporium OB3b and Escherichia coli to the library

sequences, because translation initiation region (around 30 -nt before and after the start codon) is a determinant of translational efficiency $[27,28]$. The promoter-gfp gene was integrated into the non-coding region of the genome of Methylomonas sp. DH-1 for fluorescence measurement (Fig. 2A).

The strength of the promoters varied from $0.24 \%$ to $410 \%$ in Methylomonas sp. DH-1 compared with that of the lac promoter, which shows that the library can cover approximately 1708 -fold range of expression levels in Methylomonas sp. DH-1 and can be used to fine-tune the gene expression. The transcriptional activities of the promoters are shown in Fig. 2B and Table 1. Five of the 38 evaluated promoters displayed no detectable fluorescence at all. We also found that the five promoters identified from 2D-PAGE analysis exhibited high transcriptional activity. In particular, the promoter of the mxaF gene from Methylomonas sp. DH-1 showed the highest expression level, indicating that this promoter can be used for the overexpression of a target gene. Because the mxaF gene encodes the $\alpha$-subunit of methanol dehydrogenase, a key enzyme in the methanol-to-formaldehyde conversion for methane utilization, the promoter could be highly active. In contrast, the promoter strength of the mxaF gene from $M$. trichosporium OB3b-a strong promoter [29]-was $<1 \%$ in Methylomonas sp. DH-1. The GFP intensity under the control of $\mathrm{P}_{\text {tal }}$ showed the second-highest intensity. Typically, transaldolases function in the non-oxidative phase of the PP pathway in carbohydrate metabolism to generate nicotinamide adenine dinucleotide phosphate (NADPH) and ribose, which are essential for the biosynthesis of secondary metabolites and amino acids.

In general, there is an overall correlation between transcript level and protein level, but there are also many reports on the inconsistency of the correlation [30]. For example, highly transcribed mRNAs often failed to be highly translated [31]. To investigate if the high expression of the proteins was due to the high strength of their promoters, not high efficiency in translation, we confirmed their transcript levels by using the previous transcript data of Methylomonas sp. DH-1 [11]. According to the previous gene expression profile, of the five highly expressed proteins identified by the 2D-PAGE, 
A

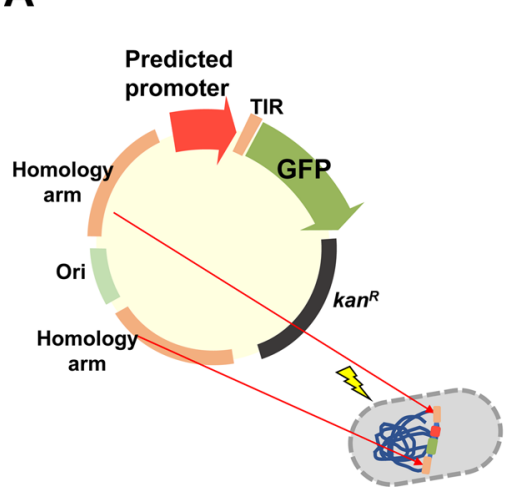

B

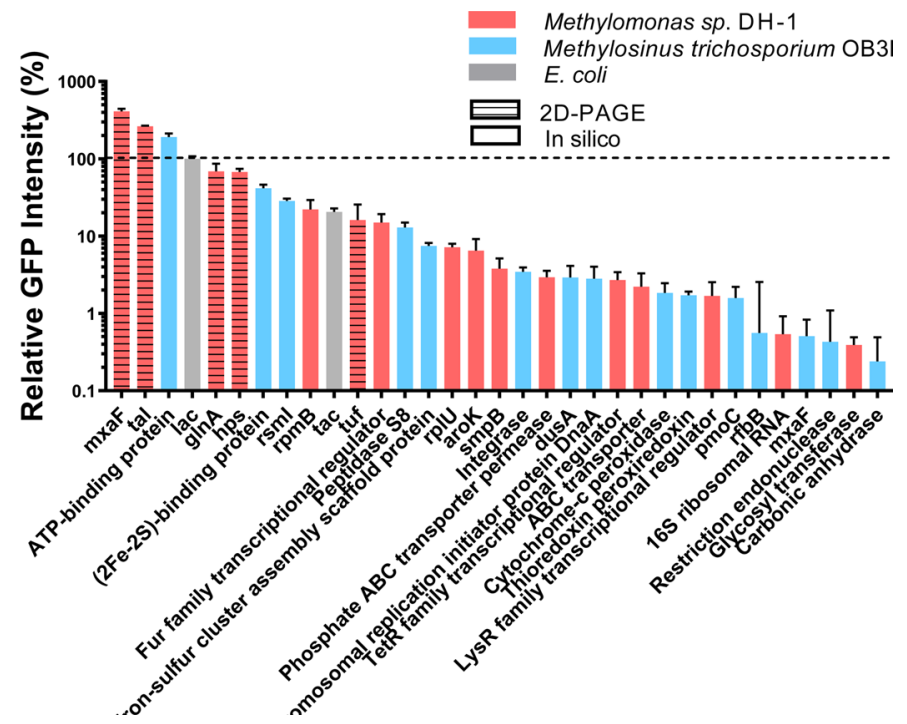

Fig. 2 Strength of promoter candidates measured by a GFP reporter. A Construction of a GFP expression plasmid under the control of a predicted promoter for integration into a Methylomonas sp. DH-1 chromosome. B Promoter strength measurement in Methylomonas sp. DH-1 by flow cytometry. Promoters showed up to 410-fold differences in GFP expression. The strength was normalized by that of the lac promoter (100\%). As the $y$-axis is drawn at log scale, five promoter candidates that showed no detectable fluorescence were excluded from the graph. Data indicate mean $\pm \operatorname{SEM}(n=3)$

only the transcription levels of three proteins were available (methanol dehydrogenase, transaldolase, and 3-hexulose-6-phosphate synthase). The three proteins were involved in the methanol oxidation, the PP pathway, and the RuMP cycle, respectively, and were grouped into a high transcription category (Fig. 2B). Interestingly, the GFP intensities expressed from the $\mathrm{P}_{\text {ATP-binding protein }}$ of $M$. trichosporium OB3b and the lac promoter of E. coli took third and fourth places, respectively. Because of the wide range of expression levels, the developed library of promoters can be utilized for fine-tuning gene expression in Methylomonas sp. DH-1. It should be noted that promoters may display different activity under different conditions due to regulatory effects. Thus, for the consistent measurement of promoter activity, we chose a defined expression context, such as exponential growth phase and methane as a carbon source [32], and the developed library was ensured to operate under the defined context.

\section{Influence of the expression level of cadA on cell fitness and cadaverine production}

Because Methylomonas sp. DH-1 has various metabolic pathways, such as the RuMP pathway, the PP pathway, the EMP pathway, the TCA cycle, and the serine cycle, it has been engineered as a promising host platform for C1 microbial cell factories [12-16]. As a practical application of our tunable promoter library, we diversified the expression level of the cadA gene to maximize the cadaverine titre from Methylomonas sp. DH-1.
Generally, the microbial production of cadaverine, a natural nylon monomer used for polyamide production, is achieved through the overexpression of the E. coli lysine decarboxylase (CadA), which bioconverts lysine to cadaverine. Hence, it can be used in the chemical industry $[33,34]$. The biosynthetic pathway towards lysine/ cadaverine synthesis in Methylomonas sp. DH-1 is illustrated in Fig. 3. In native Methylomonas sp. DH-1, lysine synthesis involves oxaloacetate (OAA) derived from the serine and TCA cycles. However, because of the lack of the $\operatorname{cad} A$ gene, the bacterium cannot synthesize cadaverine. Thus, we introduced the cadA gene from $E$. coli into the genome of Methylomonas sp. DH-1 for cadaverine production.

Fine-tuning the CadA expression is important for efficient cadaverine production and cell fitness, because overexpressed CadA might deplete lysine, which is also used as a precursor for cell wall synthesis [35]. In addition, the highly accumulated cadaverine could inhibit the activity of CadA [36] or induce cytotoxicity. Thus, the level of CadA expression should be balanced for optimum cell growth/toxicity and cadaverine production.

We employed five promoters of different strengths from our library to diversify the expression level of CadA (Fig. 4A). The promoters and their relative strengths were $\mathrm{P}_{\text {DnaA }}(2.81 \%), \mathrm{P}_{\text {Integrase }}(3.45 \%), \mathrm{P}_{\text {rpmB }}(22.09 \%)$, $\mathrm{P}_{(2 \mathrm{Fe}-2 \mathrm{~S}) \text {-binding protein }}(41.54 \%)$, and $\mathrm{P}_{\mathrm{mxaF}}(411 \%)$. We then introduced the five constructed genes into the genome of Methylomonas sp. DH-1 and tested the resulting strains 
Table 1 Tunable promoter library for Methylomonas sp. DH-1

\begin{tabular}{|c|c|c|}
\hline Promoter identification method & Gene name & $\begin{array}{l}\text { Promoter } \\
\text { strength } \\
\text { (\%) }\end{array}$ \\
\hline \multirow[t]{15}{*}{ In silico prediction (Methylomonas sp. DH-1) } & $50 S$ ribosomal protein L28 (rpmB) & 22.09 \\
\hline & Fur family transcriptional regulator & 14.99 \\
\hline & $50 S$ ribosomal protein L21 (rp/U) & 7.16 \\
\hline & Shikimate kinase (aroK) & 6.49 \\
\hline & SsrA-binding protein $(s m p B)$ & 3.79 \\
\hline & Phosphate $A B C$ transporter permease & 2.94 \\
\hline & TetR family transcriptional regulator & 2.7 \\
\hline & $\mathrm{ABC}$ transporter & 2.21 \\
\hline & LysR family transcriptional regulator & 1.68 \\
\hline & $16 \mathrm{~S}$ ribosomal RNA & 0.54 \\
\hline & Glycosyl transferase & 0.39 \\
\hline & Cytochrome c oxidase subunit 2 & 0.0 \\
\hline & Elongation factor $\mathrm{P}(e f p)$ & 0.0 \\
\hline & $50 S$ ribosomal protein L31 (rpmE) & 0.0 \\
\hline & 10 kDa chaperonin (gros) & 0.0 \\
\hline \multirow[t]{14}{*}{ In silico prediction (M. trichosporium OB3b) } & ATP-binding protein & 191.08 \\
\hline & (2Fe-2S)-binding protein & 41.54 \\
\hline & Ribosomal RNA small methyltransferase I (rsml) & 28.42 \\
\hline & Peptidase S8 & 12.92 \\
\hline & Iron-sulphur cluster assembly scaffold protein & 7.45 \\
\hline & Integrase & 3.45 \\
\hline & tRNA-dihydrouridine(20/20a) synthase (dusA) & 2.91 \\
\hline & Chromosomal replication initiator protein DnaA & 2.81 \\
\hline & Cytochrome $c$ peroxidase & 1.84 \\
\hline & Thioredoxin peroxiredoxin & 1.71 \\
\hline & dTDP-glucose 4,6-dehydratase $(r f b B)$ & 0.56 \\
\hline & Restriction endonuclease & 0.43 \\
\hline & Carbonic anhydrase & 0.24 \\
\hline & GGDEF domain-containing protein & 0.0 \\
\hline \multirow[t]{5}{*}{ 2D-PAGE (Methylomonas sp. DH-1) } & Methanol dehydrogenase (mxaF) & 411.18 \\
\hline & Transaldolase (tal) & 262.9 \\
\hline & Glutamine synthetase $(g \ln A)$ & 68.74 \\
\hline & 3-hexulose-6-phosphate synthase (hps) & 67.37 \\
\hline & Elongation factor Tu (tuf) & 16.12 \\
\hline \multirow[t]{2}{*}{ M. trichosporium OB3b promoters } & Methane monooxygenase ( $p m o C$ ) promoter $C$ & 1.58 \\
\hline & Methanol dehydrogenase (mxaF) promoter & 0.51 \\
\hline \multirow[t]{2}{*}{ E. coli promoters } & lac promoter (without operator) & 100 \\
\hline & tac promoter (without operator) & 20.54 \\
\hline
\end{tabular}

for the desired bioconversion in shake-flask cultures with a supplementation of $30 \%$ methane $(\mathrm{v} / \mathrm{v})$ as the sole carbon source. Therefore, we measured cadaverine and its precursor lysine titres along with cell growth. As shown in Fig. 4B, C, the CadA overexpression by two strong promoters $\left(\mathrm{P}_{\mathrm{mxaF}}\right.$ and $\left.\mathrm{P}_{(2 \mathrm{Fe}-2 \mathrm{~S}) \text {-binding protein }}\right)$ failed to produce cadaverine and greatly retarded the cell growth (Fig. 4B, C). On the contrary, the strains expressing CadA under the control of three weeks or moderate promoters $\left(\mathrm{P}_{\text {DnaA }}, \mathrm{P}_{\text {Integrase, }}\right.$ and $\left.\mathrm{P}_{\mathrm{rpmB}}\right)$ displayed normal growth and higher cadaverine titres of $2.85 \pm 0.13,11.55 \pm 2.70$, and $8.32 \pm 0.81 \mathrm{mg} / \mathrm{L}$, respectively, after $72 \mathrm{~h}$ of cultivation. In a previous study, the same tendency of cadaverine titre change with respect to the level of CadA had been observed in E. coli [37]: when CadA was highly expressed, the cadaverine titre was decreased. One 


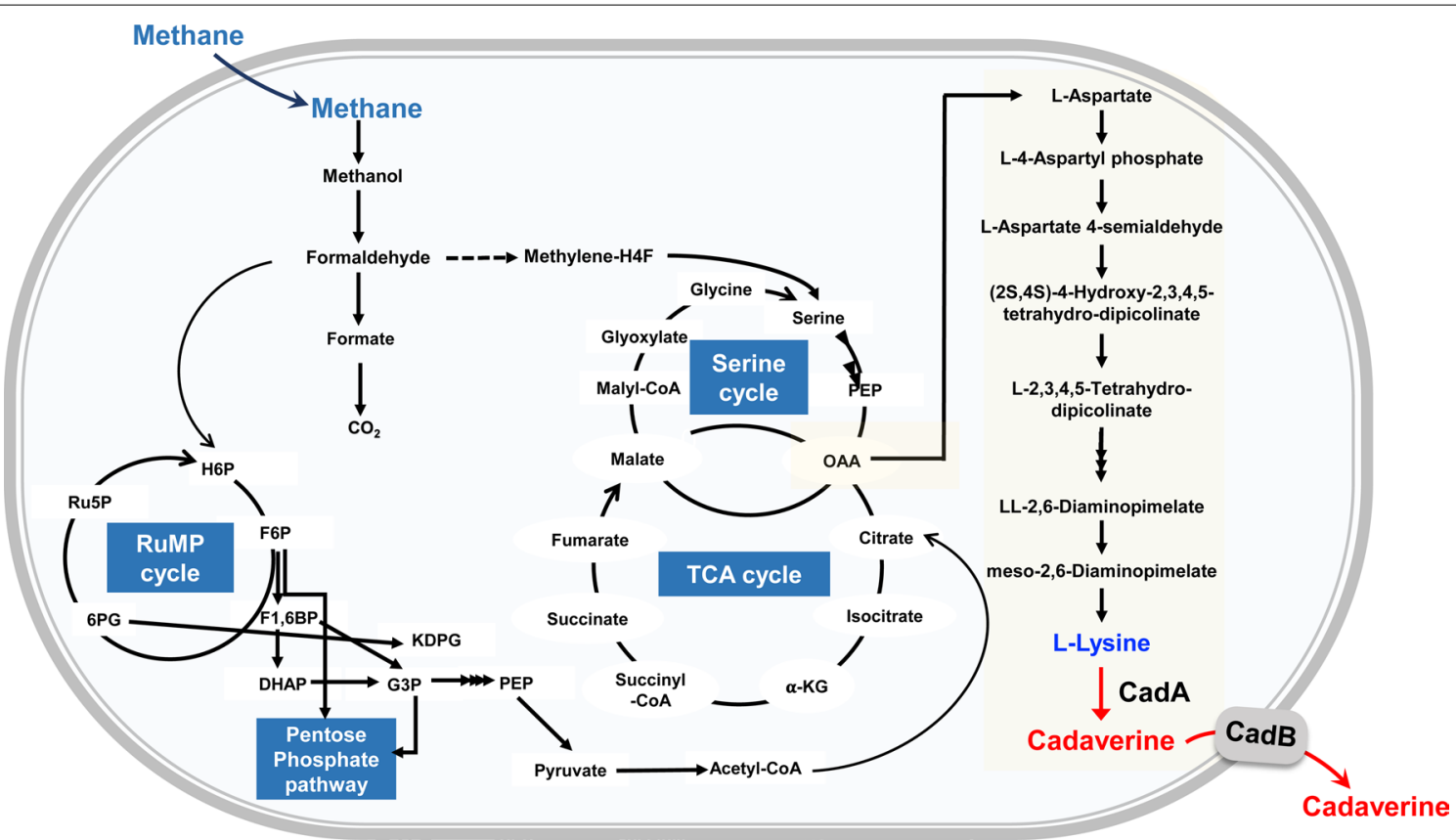

Fig. 3 Metabolic pathways of Methylomonas sp. DH-1 for the production of cadaverine from methane. Reactions mediated by heterologous enzymes are shown in red. CadA, L-lysine decarboxylase; CadB, lysine/cadaverine antiporter protein

plausible hypothesis for the decreased cadaverine titre is lysine depletion. As shown in Fig. 4D, when the two strong promoters were employed, the lysine titre inside Methylomonas sp. DH-1 was remarkably decreased. Since lysine is an important amino acid for growth, the depletion also induced cell growth defect [38], which in turn resulted in a decrease in overall cadaverine titre (Fig. 4B). Interestingly, the lysine concentrations in the strains of the three weak or moderate promoters were not statistically different from that of wild type when tested by $\mathrm{t}$-test ( $p$-value $>0.1)$, even though the lysine-consuming cadA gene was introduced. It is thus likely that CadA induced a metabolic flux in the lysine biosynthetic pathway by consuming lysine at an appropriate rate. Collectively, these results suggest that fine-tuning the expression of a target gene, rather than its overexpression, is a key to engineer metabolic pathways and that our tunable library can provide a genetic tool for optimization.

\section{Improved cadaverine production by optimizing cadB expression}

The optimization of $\operatorname{cad} A$ gene expression in Methylomonas sp. DH-1 using a promoter library helped enhance cadaverine production without redesigning the lysine synthetic pathway. It has been reported that the co-expression of $\mathrm{CadA}$ and $\mathrm{CadB}$ (a lysine/cadaverine antiporter) could simultaneously enhance lysine and cadaverine production because $\mathrm{CadB}$ exports intracellular cadaverine, a feedback inhibitor of CadA [33, $35,36]$. To further enhance cadaverine production, we introduced the $c a d B$ gene into three cadaverine-producing strains, $\mathrm{P}_{\text {DnaA }}-c a d A, \mathrm{P}_{\text {Integrase }}-c a d A$, and $\mathrm{P}_{\mathrm{rpmB}}-c a d A$. To investigate whether the fine-tuning of $\mathrm{CadB}$ gene expression could also affect cell growth and lysine/cadaverine production, we co-expressed the $c a d B$ gene under three different promoters $\left(\mathrm{P}_{\mathrm{DnaA}}, \mathrm{P}_{(2 \mathrm{Fe}-2 \mathrm{~S}) \text {-binding protein }}\right.$, and $\mathrm{P}_{\text {mxaF }}$ ) and constructed nine strains in total (Fig. 5A).

As shown in Fig. $5 \mathrm{~B}$, the highest cadaverine titres, $11.18 \pm 0.07$ and $14.46 \pm 1.01 \mathrm{mg} / \mathrm{L}$, were achieved from the $\mathrm{P}_{\text {DnaA }}-c a d A / \mathrm{P}_{\text {DnaA }}-c a d B$ and $\mathrm{P}_{\mathrm{rpmB}}-c a d A / \mathrm{P}_{\text {DnaA }}-c a d B$ strains, respectively, after $72 \mathrm{~h}$ of cultivation. The cadaverine titres were improved by 3.92- and 1.73-fold compared with those of their parental strains, $\mathrm{P}_{\text {DnaA }}$-cadA and $\mathrm{P}_{\mathrm{rpmB}}-c a d A$, respectively. Interestingly, although the introduction of $c a d B$ increased the lysine titre in all $\operatorname{cad} A / \operatorname{cadB}$ knock-in strains, its introduction into the $\mathrm{P}_{\text {Integrase }}-c a d A$ strain reduced cadaverine production in all promoters. To investigate the reason for the inefficient production of cadaverine in these strains, the cell growth and cadaverine production were monitored for $120 \mathrm{~h}$ (Fig. 6A, B). Interestingly, when $c a d B$ was overexpressed, the cells showed a major growth defect and decreased the cadaverine titre. Unlike the $c a d A$ gene, the overexpression of the $c a d B$ gene did not deplete the intracellular lysine concentration (Fig. $5 \mathrm{C}$ ). One plausible explanation is that the additional overproduction of $\mathrm{CadB}$ consumed 
A

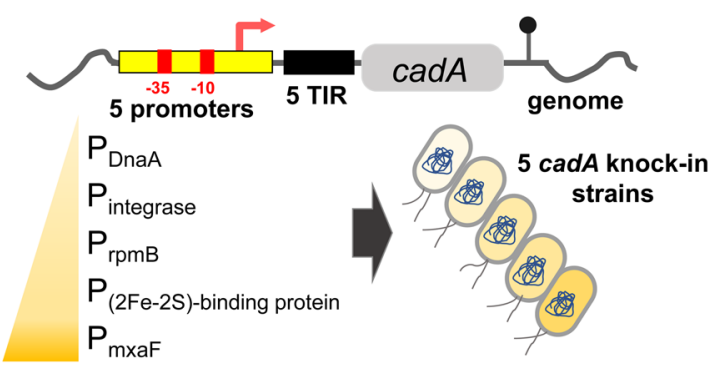

C

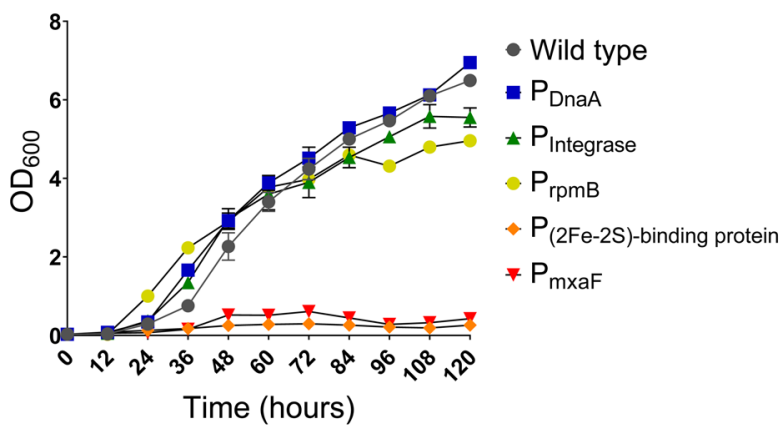

B
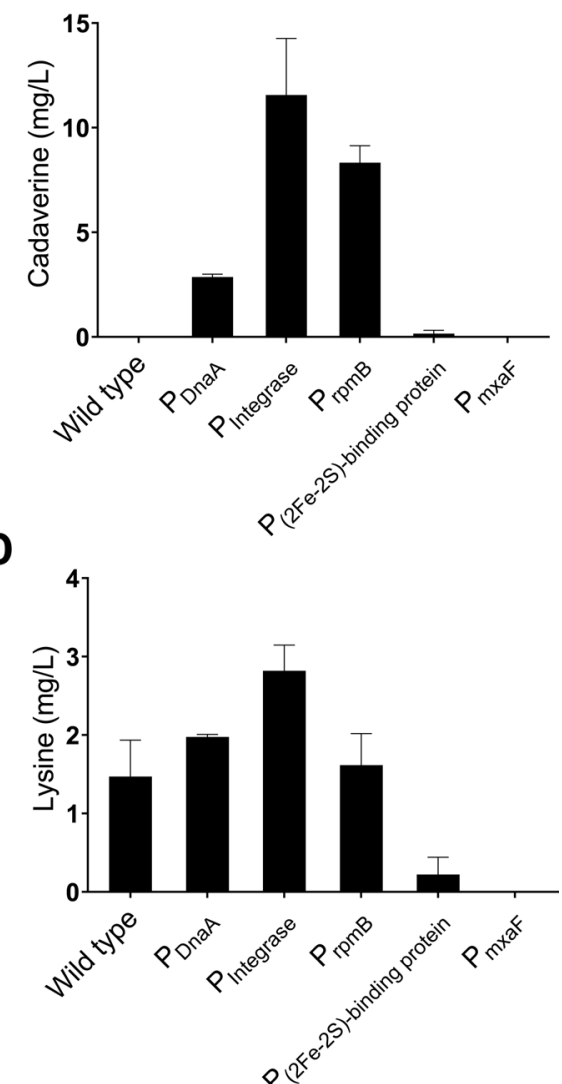

Fig. 4 Effects of the expression level of the cadA gene on cell growth and lysine/cadaverine biosynthesis in Methylomonas sp. DH-1. A Genetic structure of cadA under the control of five promoters. Cadaverine production (B), growth (C), and lysine synthesis (D) of wild-type and five engineered Methylomonas sp. DH-1 strains in an NMS medium with a supplementation of $30 \%$ methane $(\mathrm{v} / \mathrm{v})$ as the sole carbon source. Data indicate mean \pm SEM $(n=3)$

intracellular resources and posed a burden to the cell, thereby retarding the cell growth; however, this should be elucidated further. After $96 \mathrm{~h}$ of cultivation, the engineered $\mathrm{P}_{\mathrm{rpmB}}-c a d A / \mathrm{P}_{\mathrm{DnaA}}-c a d B$ strain afforded the maximum cadaverine titre $(18.12 \pm 1.06 \mathrm{mg} / \mathrm{L})$, which was 2.18 -fold higher than that $(8.32 \pm 0.81 \mathrm{mg} / \mathrm{L})$ of its parental strain $\left(\mathrm{P}_{\mathrm{rpmB}}\right.$-cadA strain) and 2.78-fold higher than that $(6.50 \pm 0.02 \mathrm{mg} / \mathrm{L})$ of the non-optimized $c a d A / c a d B$ strain $\left(\mathrm{P}_{\mathrm{rpmB}}-c a d A / \mathrm{P}_{(2 \mathrm{Fe}-2 \mathrm{~S}) \text {-binding protein }}-c a d B\right)$.

\section{Conclusions}

For the first time, a tunable library consisting of 33 promoters of different strengths was constructed for the gene expression optimization of Methylomonas sp. DH-1. The results showed that the fine-tuning of transcription rather than overexpression allows efficient production of recombinant proteins and regulation of metabolic pathways in cells. In future studies, an inducible system based on the tunable promoter library should be designed for more dynamic control of transcription. This system will help make methanotrophs a major sustainable platform for producing value-added products via $\mathrm{C} 1$ assimilation. Overall, the promoter library discussed here will facilitate the genetic manipulation of Methylomonas sp. DH-1 for successful implementation of methanotroph biotechnology.

\section{Methods}

\section{Growth conditions}

The E. coli DH5 $\alpha$ strain was utilized for DNA cloning and plasmid preparation. A lysogeny broth (LB) medium, containing $10 \mathrm{~g}$ of tryptone, $5 \mathrm{~g}$ of yeast extract, and $10 \mathrm{~g}$ of sodium chloride per litre, was used for the cultivation of the E. coli strain, with appropriate antibiotics $(100 \mu \mathrm{g} /$ $\mathrm{mL}$ of ampicillin or $50 \mu \mathrm{g} / \mathrm{mL}$ of kanamycin).

Methylomonas sp. DH-1 (KCTC13004BP) was used as a parental strain of the engineered strains. It was cultured in a nitrate mineral salt (NMS) medium [14] supplemented with $30 \%(\mathrm{v} / \mathrm{v})$ methane at $30{ }^{\circ} \mathrm{C}$ with shaking at $250 \mathrm{rpm}$. For genetic integration, the cells 
A

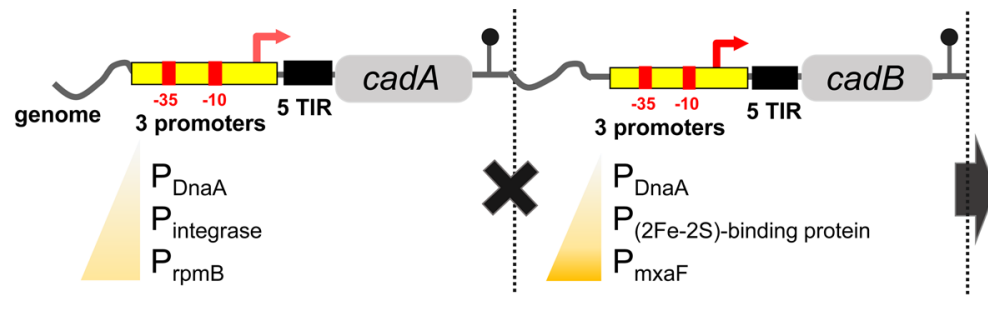

9 cadA/cadB knock-in strains

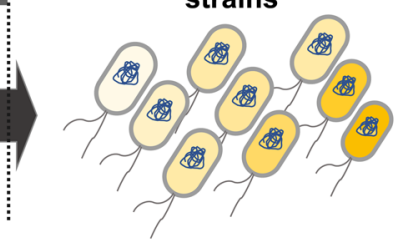

B

C
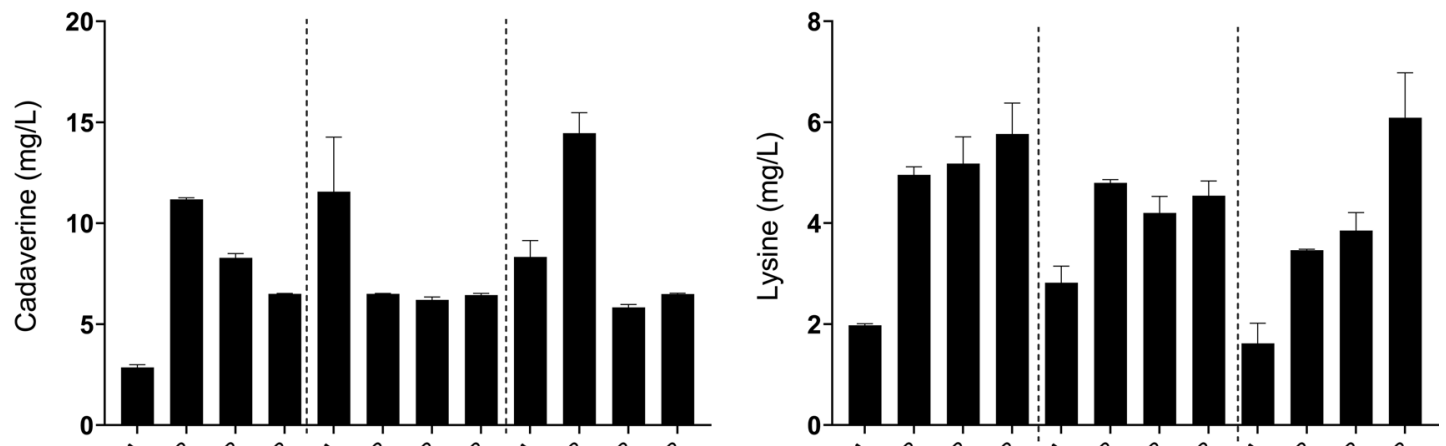

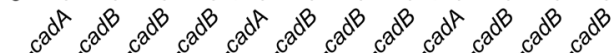

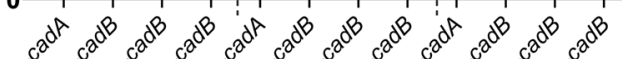

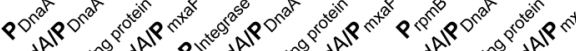
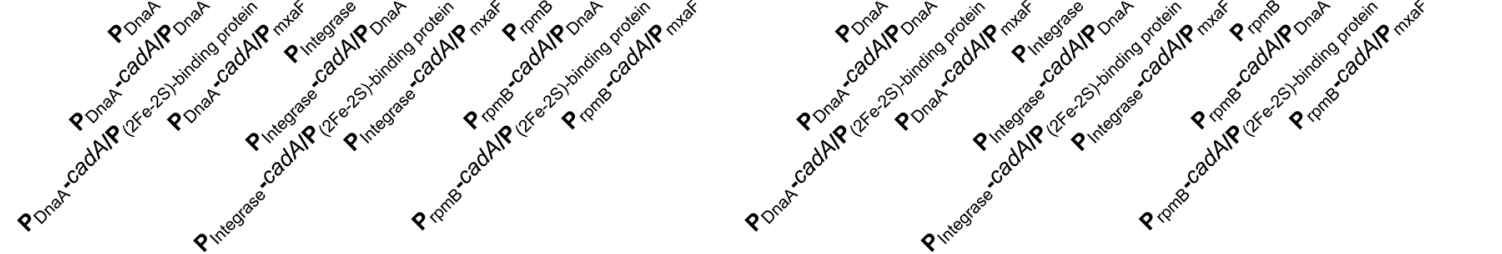

Fig. 5 Effect of the expression level of the cadB gene on lysine/cadaverine biosynthesis in three selected cadA knock-in strains. A Genetic structure for cadA and cadB expression. Three different promoters ( $\mathrm{P}_{\text {DnaA }}, \mathrm{P}_{(2 \mathrm{Fe}-25) \text {-binding protein, }}$ and $\left.\mathrm{P}_{\mathrm{mxaF}}\right)$ were employed to diversify the expression level of cadB. Cadaverine (B) and lysine titres (C) in the engineered Methylomonas sp. DH-1 strains. Data indicate mean $\pm \operatorname{SEM}(n=3)$

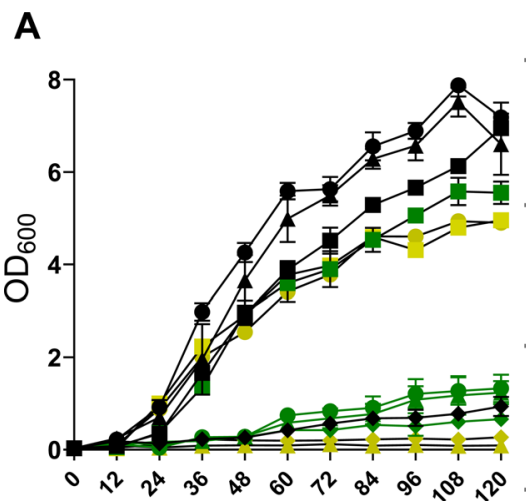

Time (hours)
B

- $\mathrm{P}_{\text {DnaA }}-\mathrm{cadA}$

- $P_{\text {DnaA }}-c a d A / P_{\text {DnaA }}-c a d B$

$\star \mathrm{P}_{\mathrm{DnaA}}-\mathrm{cadA} / \mathrm{P}_{(2 \mathrm{Fe}-2 \mathrm{~S}) \text {-binding protein }}-\mathrm{cadB}$

- $P_{\text {DnaA }}-c a d A / P_{\text {mxaF }}-c a d B$

- PIntegrase - cadA

- $P_{\text {Integrase }}-c a d A / P_{\text {DnaA }}-c a d B$

* $P_{\text {Integrase }}-$ cadA/P $P_{(2 \mathrm{Fe}-2 S) \text {-binding protein }}-\mathrm{Cad} B$

- PIntegrase-cadA/P mxaF- $^{-c a d B}$

- $P_{\text {rpmB }}-$ cadA

- $P_{\text {rpmB }}-c a d A / P_{D n a A}-c a d B$

- $\mathrm{P}_{\mathrm{rpmB}}-\mathrm{cadA} / \mathrm{P}_{(2 \mathrm{Fe}-2 \mathrm{~S}) \text {-binding protein }}-\mathrm{cadB}$

$\therefore P_{\text {rpmB }}-c a d A / P_{\text {mxaF }}-c a d B$

$$
\begin{array}{rccc}
24 & 48 & 72 & 96 \\
& \text { Time (hours) }
\end{array}
$$

Fig. 6 Cell growth $(\mathbf{A})$ and cadaverine titre $(\mathbf{B})$ over time with respect to the employed promoter for the cadB gene. Data indicate mean \pm SEM $(n=3)$ 
were transformed with plasmid-containing homology arms by electroporation [19] and grown in NMS plates with appropriate antibiotics $(100 \mu \mathrm{g} / \mathrm{mL}$ of ampicillin and/or $10 \mu \mathrm{g} / \mathrm{mL}$ of kanamycin). To measure cell growth, Methylomonas sp. DH-1 cells were grown until the stationary phase was reached. They were then diluted to $\mathrm{OD}_{600}=0.03$ with a fresh NMS medium and $30 \%(\mathrm{v} / \mathrm{v})$ methane. For repeat methane feeding, gas substitution was performed using a gas-tight syringe. The headspace was refreshed daily. Cell growth $\left(\mathrm{OD}_{600}\right)$ was monitored for 5 days using a Hitachi U5100 UVVis spectrophotometer (Tokyo, Japan).

\section{Computational prediction of methanotroph promoters}

We obtained whole genome sequences and gene annotation data of two bacteria, Methylomonas sp. DH-1 (CP014360.1) and M. trichosporium Ob3b (CP023737.1), from NCBI GenBank. From the chromosome data, we collected 100-bp upstream region from the transcription start site (TSS) of all genes as potential promoter sequences, except for the genes that produce a hypothetical protein.

To identify the methanotroph promoter, we employed two promoter prediction models: Softberry BPROM [24] and the BDGP neural network promoter prediction tool [23]. To increase the prediction accuracy, we selected the promoter regions recognized by both tools as candidates. As a result, 93 sequences from Methylomonas sp. DH-1 and 17 from M. trichosporium Ob3b were predicted as methanotroph promoter candidates.

\section{D-PAGE experiments}

2D-PAGE experiments were performed as described previously [39]. The Methylomonas sp. DH-1 cells were suspended and mixed with a lysis buffer $(8 \mathrm{M}$ urea, $2 \mathrm{M}$ thiourea, $40 \mathrm{mM}$ Tris, $65 \mathrm{mM}$ DTT, and $4 \%$ $(\mathrm{w} / \mathrm{v})$ CHAPS). Proteins in the supernatant $(200 \mu \mathrm{g})$ were diluted into $340 \mu \mathrm{L}$ of a rehydration buffer $(8 \mathrm{M}$ urea, $2 \mathrm{M}$ thiourea, $20 \mathrm{mM}$ DTT, $2 \%(\mathrm{w} / \mathrm{v})$ CHAPS, $0.8 \%(\mathrm{w} / \mathrm{v})$ immobilized $\mathrm{pH}$ gradient (IPG) buffer, and $1 \%(\mathrm{v} / \mathrm{v})$ cocktail protease inhibitor) and then loaded onto Immobiline DryStrip gels $(18 \mathrm{~cm}, \mathrm{pH} 3-10 \mathrm{NL}$; GE Healthcare Bio-Sciences, Uppasala, Sweden). The loaded IPG strips were rehydrated, focused, and equilibrated and then transferred to $12 \%(\mathrm{w} / \mathrm{v})$ SDS-polyacrylamide gels. The 2D image was analysed using PDQuest 2D Analysis Software (BioRad). The protein spots in the 2D-gel were identified by comparing them with those of a previous report and using the E. coli 2D database (http://world-2dpage.expasy.org/swiss2 dpage/viewer) as a reference [26].

\section{Construction of plasmids for genomic integration into Methylomonas sp. DH-1}

The pIns plasmid [19], which carries two 1-kb-long homology arms for genomic integration into the genome of Methylomonas sp. DH-1 and the resistance marker gene $\mathrm{Kan}^{\mathrm{R}}$, was used to develop further plasmid versions. To measure the strength of the predicted promoters, we additionally inserted the predicted promoter sequence, a modified TIR sequence, and a $g f p$ gene between the two homology arms through restriction enzyme digestion and ligation. The promoter sequences were amplified from the genomic DNA of Methylomonas sp. DH-1. The correct insertion was verified by DNA sequencing. The plasmid construction is shown in Fig. 2A.

To integrate lysine decarboxylase gene $\operatorname{cad} A$ for cadaverine production, the coding sequence was amplified from E. coli W3110 genomic DNA and cloned into the plns plasmid instead of the $g f p$ gene under the control of five selected promoters. In addition, for double integration of cadaverine transport protein gene $c a d B$ in the background of the cadA knock-in cells, the homologous regions of the plns plasmid were modified to insert $c a d B$ at a different genomic location and the resistance gene was changed to $A m p^{\mathrm{R}}$. Then, the coding sequence of $c a d B$ was amplified from $E$. coli W3110 genomic DNA and cloned into the $c a d A$ locus under the control of three selected promoters. Each generated plasmid was integrated into a non-coding region of the Methylomonas sp. DH-1 chromosome for genetic manipulation by homologous recombination. All plasmids and strains used in this study are listed in Additional file 5: Table S4.

\section{Flow cytometry}

To measure the promoter strength, the $g f p$ knock-in cells were prepared in the exponential phase. Then, the cells were diluted to $\mathrm{OD}_{600}=0.1$ with $1 \times$ phosphate-buffered saline (PBS), and the fluorescence intensity was quantitatively measured using a Guava EasyCyte flow cytometer (Millipore, Darmstadt, Germany). A total of 50,000 events were recorded to determine GFP fluorescence at a flow rate of $0.12 \mu \mathrm{L} / \mathrm{s}$. The excitation was conducted by a 488-nm laser and the emission light was collected with a 525/30-nm bandpass filter.

\section{High-performance liquid chromatography (HPLC)}

To measure cadaverine and L-lysine concentrations, 500 $\mu \mathrm{L}$ of culture supernatants was filtered through a 0.22 $\mu \mathrm{m}$ syringe filter and analysed by HPLC, as previously reported [40]. In brief, cadaverine and L-lysine concentrations were measured by precolumn $o$-phthaldialdehyde 
derivatization coupled with reverse-phase HPLC and UV detection. The derivatized cadaverine and L-lysine were detected by a variable wavelength detector at $230 \mathrm{~nm}$.

\section{Statistics and reproducibility}

Data obtained from at least three independent experiments were analysed using GraphPad Prism v7.0 (GraphPad Software, Inc.). The replicates were plotted using the average and standard error of the mean (SEM).

\begin{abstract}
Abbreviations
TCA: Tricarboxylic acid; RuMP: Ribulose monophosphate; EMP: Embden-Meyerhof-Parnas; PP: Pentose phosphate (PP); EDD: Entener-Doudoroff; MEP: Methylerythritol 4-phosphate; 2D-PAGE: 2D gel electrophoresis; MALDI-TOF: Matrix-assisted laser desorption/ionization-time of flight; PQQ: Pyrroloquinoline quinone; mxaF: Methanol dehydrogenase; glnA: Glutamine synthetase; tuf: Elongation factor Tu; tal: Transaldolase; hps: 3-Hexulose-6-phosphate synthase; pmoC: Methane monooxygenase; gfp: Green fluorescent protein; NADPH: Nicotinamide adenine dinucleotide phosphate; CadA: Lysine decarboxylase; OAA: Oxaloacetate; CadB: Lysine/cadaverine antiporter; NMS: Nitrate mineral salt; TSS: Transcription start site; PBS: Phosphate-buffered saline.
\end{abstract}

\section{Supplementary Information}

The online version contains supplementary material available at https://doi. org/10.1186/s13068-021-02077-8.

Additional file 1: Fig S1. Total proteome of Methylomonas sp. DH-1 analyzed by 2D-PAGE. Five high-density protein spots are indicated by arrows and identified protein names are listed on the right.

Additional file 2: Table S1.Predicted promoter sequences from the genome of Methylomonas sp. DH-1.

Additional file 3: Table S2. Predicted promoter sequences from the genome of M. trichosporium OB3b.

Additional file 4: Table S3. Five promoter sequences identified from 2D-PAGE of Methylomonas sp. DH-1.

Additional file 5: Table S4. Bacterial strains and plasmids used in this study.

\section{Acknowledgements}

Not applicable.

\section{Authors' contributions}

DN supervised this study and revised the manuscript. HL carried out the transformation of Methylomonas sp. DH-1, the FACS analysis, the HPLC analysis, and drafted the manuscript. JR, HK, WK, and JS performed the construction of the plasmids. MY carried out the promoter prediction. SY and SE carried out the 2D-PAGE analysis. All the authors read and approved the final manuscript.

\section{Funding}

This work was supported by the National Research Foundation of Korea (NRF) grant funded by the Korea government (MSIT) (No. NRF-2018R1A5A1025077). This research was also supported by Basic Science Research Program through the National Research Foundation of Korea (NRF) funded by the Ministry of Education (No. 2021R1/1A1A01056588)

\section{Availability of data and materials}

Not applicable.

\section{Declarations}

Ethics approval and consent to participate

Not applicable.

\section{Consent for publication}

Not applicable.

\section{Competing interests}

The authors declare that they have no competing interests.

\section{Author details}

${ }^{1}$ Department of Biomedical Engineering, Chung-Ang University, 84 Heukseok-ro Dongjak-gu, Seoul 06974, Republic of Korea. ${ }^{2}$ Department of Life Science, Chung-Ang University, Seoul 06974, Republic of Korea.

Received: 16 September 2021 Accepted: 16 November 2021

Published online: 04 December 2021

\section{References}

1. Gažová I, Lefevre L, Bush SJ, Clohisey S, Arner E, de Hoon M, et al. The transcriptional network that controls growth arrest and macrophage differentiation in the human myeloid leukemia cell line THP-1. Front Cell Dev Biol. 2020;8(498).

2. DL Rothman SC Stearns RG Shulman 2021 Gene expression regulates metabolite homeostasis during the Crabtree effect: implications for the adaptation and evolution of metabolism Proc Natl Acad Sci USA 1182 e2014013118

3. S Roy TT Saha L Johnson B Zhao J Ha KP White 2015 Regulation of gene expression patterns in mosquito reproduction PLoS Genet 118 e1005450

4. YS Michaels MB Barnkob H Barbosa TA Baeumler MK Thompson V Andre 2019 Precise tuning of gene expression levels in mammalian cells Nat Commun 101818

5. Z Lu S Yang X Yuan Y Shi L Ouyang S Jiang 2019 CRISPR-assisted multidimensional regulation for fine-tuning gene expression in Bacillus subtilis Nucleic Acids Res 477 e40

6. H-M Lee PNL Vo D Na 2018 Advancement of metabolic engineering assisted by synthetic biology Catalysts 812619

7. J Ren J Lee D Na 2020 Recent advances in genetic engineering tools based on synthetic biology J Microbiol 581110

8. C Troein D Ahren M Krogh C Peterson 2007 Is transcriptional regulation of metabolic pathways an optimal strategy for fitness? PLoS ONE 29 e855

9. R Santibanez D Garrido AJM Martin 2020 Atlas: Automatic modeling of regulation of bacterial gene expression and metabolism using rule-based languages Bioinform 365473

10. AD Nguyen IY Hwang OK Lee DH Hur YC Jeon S Hadiyati 2018 Functional analysis of Methylomonas sp. DH-1 genome as a promising biocatalyst for bioconversion of methane to valuable chemicals Catalysts 83117

11. AD Nguyen D Kim EY Lee 2019 A comparative transcriptome analysis of the novel obligate methanotroph Methylomonas sp. DH-1 reveals key differences in transcriptional responses in C1 and secondary metabolite pathways during growth on methane and methanol BMC Genomics 201 130

12. DH Hur J-G Na EY Lee 2017 Highly efficient bioconversion of methane to methanol using a novel type I Methylomonas sp. DH-1 newly isolated from brewery waste sludge J Chem Technol Biotechnol 922311318

13. TT Nguyen IY Hwang JG Na EY Lee 2019 Biological conversion of propane to 2-propanol using group I and II methanotrophs as biocatalysts J Ind Microbiol 465675685

14. DTN Nguyen OK Lee S Hadiyati AN Affifah MS Kim EY Lee 2019 Metabolic engineering of the type I methanotroph Methylomonas sp. DH-1 for production of succinate from methane Metab Eng 54170179

15. JK Lee S Kim W Kim S Kim S Cha H Moon 2019 Efficient production of d-lactate from methane in a lactate-tolerant strain of Methylomonas sp. $\mathrm{DH}-1$ generated by adaptive laboratory evolution Biotechnol Biofuels 12 234 
16. DH Hur TT Nguyen D Kim EY Lee 2017 Selective bio-oxidation of propane to acetone using methane-oxidizing Methylomonas sp DH-1 J Ind Microbiol Biotechnol 44710971105

17. J Ren D Na SM Yoo 2018 Optimization of chemico-physical transformation methods for various bacterial species using diverse chemical compounds and nanomaterials J Biotechnol 2885560

18. J Ren H-M Lee TD Thai D Na 2020 Identification of a cytosine methyltransferase that improves transformation efficiency in Methylomonas sp. DH-1 Biotechnol Biofuels 131200

19. H-M Lee J Ren KM Tran B-M Jeon W-U Park H Kim 2021 Identification of efficient prokaryotic cell-penetrating peptides with applications in bacterial biotechnology Commun Biol 41205

20. E Balleza LN Lopez-Bojorquez A Martinez-Antonio O Resendis-Antonio I Lozada-Chavez YI Balderas-Martinez 2009 Regulation by transcription factors in bacteria: beyond description FEMS Microbiol Rev 331133151

21. B Desvergne L Michalik W Wahli 2006 Transcriptional regulation of metabolism Physiol Rev 862465514

22. NM Belliveau SL Barnes WT Ireland DL Jones MJ Sweredoski A Moradian 2018 Systematic approach for dissecting the molecular mechanisms of transcriptional regulation in bacteria Proc Natl Acad Sci USA 11521 E4796 E4805

23. MG Reese 2001 Application of a time-delay neural network to promoter annotation in the Drosophila melanogaster genome Comput Chem 261 5156

24. Salamov VSA, Solovyevand AJM, Its Applications in Agriculture B, Studies E, Li R, editor. Automatic annotation of microbial genomes and metagenomic sequences. 2011:61-78.

25. Matsen J, Yang S, Stein L, Beck D, Kalyuzhanaya M. Global molecular analyses of methane metabolism in methanotrophic alphaproteobacterium, Methylosinus trichosporium OB3b. Part I: Transcriptomic Study. Front Microbiol. 2013;4(40)

26. L Tonella BJ Walsh JC Sanchez K Ou MR Wilkins M Tyler 1998 '98 Escherichia coli SWISS-2DPAGE database update Electrophoresis 19111960 1971

27. HM Salis EA Mirsky CA Voigt 2009 Automated design of synthetic ribosome binding sites to control protein expression Nat Biotechnol 2710 946950

28. D Na D Lee 2010 RBSDesigner: software for designing synthetic ribosome binding sites that yields a desired level of protein expression Bioinform 262026332634

29. J Yang CT Zhang XJ Yuan M Zhang XH Mo LL Tan 2018 Metabolic engineering of Methylobacterium extorquens AM1 for the production of butadiene precursor Microb Cell Fact 171194

30. Y Liu A Beyer R Aebersold 2016 On the dependency of cellular protein levels on mRNA abundance Cell 1653535550

31. Reeve B, Hargest T, Gilbert C, Ellis T. Predicting translation initiation rates for designing synthetic biology. Front Bioeng Biotechnol. 2014;2(1).

32. A Reider Apel L d'Espaux M Wehrs D Sachs RA Li GJ Tong 2016 A Cas9based toolkit to program gene expression in Saccharomyces cerevisiae Nucleic Acids Res 451496508

33. W Ma K Chen Y Li N Hao X Wang P Ouyang 2017 Advances in cadaverine bacterial production and its applications Engineering 33308317

34. J Mi S Liu H Qi J Huang X Lan L Zhang 2021 Cellular engineering and biocatalysis strategies toward sustainable cadaverine production: State of the art and perspectives ACS Sustain Chem Eng 9310611072

35. TT Nguyen OK Lee S Naizabekov EY Lee 2020 Bioconversion of methane to cadaverine and lysine using an engineered type II methanotroph, Methylosinus trichosporium OB3b Green Chem 222278037811

36. M Li D Li Y Huang M Liu H Wang Q Tang 2014 Improving the secretion of cadaverine in Corynebacterium glutamicum by cadaverine-lysine antiporter J Ind Microbiol Biotechnol 414701709

37. SM Yoo SW Jung J Yeom SY Lee D Na 2020 Tunable gene expression system independent of downstream coding sequence ACS Synth Biol 9 1129983007

38. X Li SC Ricke 2003 Generation of an Escherichia coli lys A targeted deletion mutant by double cross-over recombination for potential use in a bacterial growth-based lysine assay Lett Appl Microbiol 376458462

39. C Vijayendran S Burgemeister K Friehs K Niehaus E Flaschel 2007 2DBase: 2D-PAGE database of Escherichia coli Biochem Biophys Res Commun 363 3822827
40. ZG Qian XX Xia SY Lee 2011 Metabolic engineering of Escherichia coli for the production of cadaverine: a five carbon diamine Biotechnol Bioeng 108193103

\section{Publisher's Note}

Springer Nature remains neutral with regard to jurisdictional claims in published maps and institutional affiliations.
Ready to submit your research? Choose BMC and benefit from:

- fast, convenient online submission

- thorough peer review by experienced researchers in your field

- rapid publication on acceptance

- support for research data, including large and complex data types

- gold Open Access which fosters wider collaboration and increased citations

- maximum visibility for your research: over $100 \mathrm{M}$ website views per year

At BMC, research is always in progress.

Learn more biomedcentral.com/submissions 